$17^{\text {th }}$ International Congress of Metrology, 12003 (2015)

DOI: $10.1051 /$ metrology $/ 201512003$

(C) Owned by the authors, published by EDP Sciences, 2015

\title{
IMPROVEMENT OF THE MEASUREMENT CHAIN LINKING THE FARAD TO THE OHM AT LNE
}

Ralph, Sindjui ${ }^{1, a}$; Olivier, Thévenot ${ }^{1}$; Pierre, Gournay ${ }^{2}$; François, Piquemal ${ }^{1}$; Gael, Thuillier ${ }^{1}$; Saîf, Khan $^{1}$ et Olivier, $^{-}$ Séron ${ }^{1}$

${ }^{1}$ Laboratoire National de Métrologie et d'Essais (LNE), 29 avenue Roger Hennequin 78197 Trappes Cedex, France

${ }^{2}$ Bureau International des Poids et Mesures (BIPM), Pavillon de Breteuil, 12 bis Grande Rue 92310 Sèvres, France

\begin{abstract}
Résumé. Cet article présente l'amélioration de la chaîne de mesure du LNE permettant la réalisation de l'ohm à partir du farad. Celle-ci est mise en œuvre afin de déterminer le quantum de résistance $R_{\mathrm{K}}$ issu de l'effet Hall quantique à partir d'un condensateur calculable permettant la réalisation du farad dans le SI. Elle comporte différents ponts d'impédances coaxiaux en deux et quatre paires de bornes dont l'élément central est un transformateur étalon. Nous décrivons dans cet article une nouvelle génération d'autotransformateurs étalons double étage conçus et caractérisés dans le but d'améliorer la chaîne de mesure d'impédance du LNE et réduire l'incertitude globale sur la détermination de $R_{\mathrm{K}}$ à une valeur proche de $10^{-8}$.
\end{abstract}

\section{Introduction}

As a part of the redefinition of the International System of Units (SI) based on fundamental constants [1], the determination of the von Klitzing constant $R_{\mathrm{K}}$ with an uncertainty of one part in $10^{8}$ presents some interest.

Indeed, the comparison of the SI values of $R_{\mathrm{K}}$ obtained from direct determinations with other accurate measurements of $h / e^{2}$ serves as a relevant test of validity of the quantum Hall effect theory predicting the relation $R_{\mathrm{K}}=h / e^{2}$, a decisive issue within the context of the new SI (with $h$ the planck constant and $e$ the charge of the electron).

Direct measurement of $R_{\mathrm{K}}$ is obtained by linking the ohm produced from the quantum Hall effect to the farad achieved from a Thompson-Lampard calculable capacitor $[2,3]$, using a dedicated high precision measurement chain. At LNE, the last determination of $R_{\mathrm{K}}$ was conducted in 2000 with an uncertainty of 5 parts in $10^{8}$ [2]. In order to reach the target uncertainty of one part in $10^{8}$, the LNE needs to build a new calculable capacitor and to improve the associated measuring chain. This last point involves in particular the design and the characterization of new standard autotransformers which design and calibration is described below.

\section{Measurement chain}

Several coaxial impedance comparison bridges are used for the determination of $R_{\mathrm{K}}$. The overall view of the successive measurements is shown in Figure 1. The measurements are carried out at 3 angular frequencies $\omega=2500 \mathrm{rad} / \mathrm{s}, 5000 \mathrm{rad} / \mathrm{s}$ and $10000 \mathrm{rad} / \mathrm{s}$.

First, a $1 \mathrm{pF}$ capacitor is compared to the ThompsonLampard calculable capacitor with a two terminal-pair bridge, the ratio of which being adapted to the capacitance variation produced by the calculable capacitor.

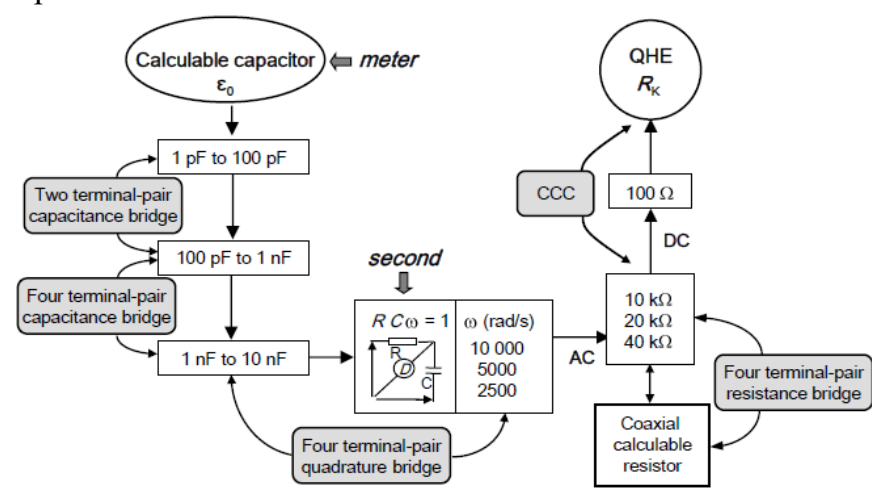

Figure 1. Measurement chain of the determination of $R_{\mathrm{K}}$

Then two 10:-1 coaxial ratio bridges (two and four terminal-pair) are used successively to link two $10 \mathrm{nF}$ capacitors (homemade invar plates in vacuum capacitors) to the calculable capacitor. The $1 \mathrm{nF}$ transfer standard is a nitrogen sealed capacitor in oil bath while the 10 and $100 \mathrm{pF}$ transfer standards are thermoregulated fused silica capacitors (Andeen-Hagerling capacitors). Next, a quadrature bridge is used to compare the impedances of the $10 \mathrm{nF}$ capacitors to that of pair of resistors. Three couples of resistors are used with values of 40, 20 and $10 \mathrm{k} \Omega$, the bridge being balanced for the three angular frequencies $2500 \mathrm{rad} / \mathrm{s}, \quad 5000 \mathrm{rad} / \mathrm{s}$ and $10000 \mathrm{rad} / \mathrm{s}$, respectively. The quadrature bridge is a 1:-1 fourterminal-pair bridge derived from the classical models described by Cutkosky [4] and Kibble [5]. Finally, taking into account their frequency dependence, by means of $\mathrm{AC} / \mathrm{DC}$ calculable resistance standards, these resistances are compared to the quantum Hall resistance standard in DC leading to a SI value of the von Klitzing constant.

aralphariel.sindjui@lne.fr 
This comparison is made using a Cryogenic Current Comparator (CCC) based resistance bridge.

Details of the implementation of these bridges are given in the reference [2]. Figure 2 hereafter shows the principle of a coaxial AC bridge for impedance comparison.

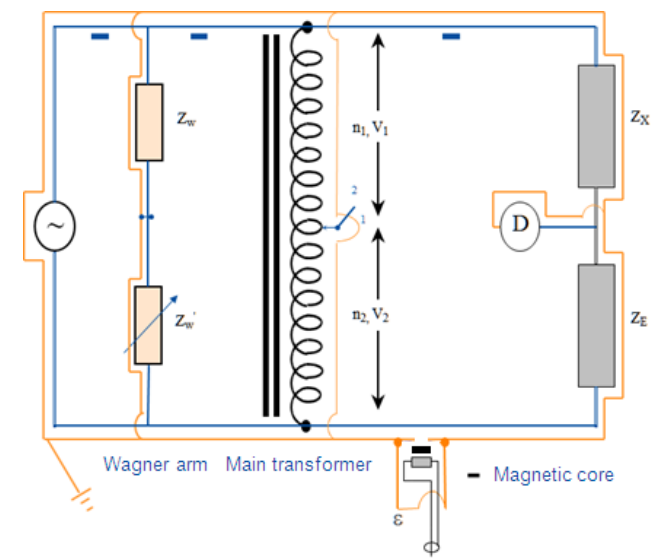

Figure 2. Principle of a coaxial $\mathrm{AC}$ bridge with $\mathrm{Z}_{\mathrm{X}}$ and $\mathrm{Z}_{\mathrm{E}}$ the impedances in comparison, $\mathrm{D}$ the detector, $\varepsilon$ the injection voltage. The Wagner arm is used to overcome the effect of the leakage impedances. The ratio of the bridge is ensured by the main transformer.

The key elements used in the 10:-1 ratio bridges are standard transformers. During the last determination of $R_{\mathrm{K}}$, the more penalizing uncertainty components associated to the measuring chain are following: the ratios of the $\mathrm{AC}$ bridges $\left(1,5.10^{-8}\right)$, the voltage effect of the capacitors $10 \mathrm{pF}, 100 \mathrm{pF}, 1000 \mathrm{pF}\left(1.10^{-8}\right)$ and the frequency dependence of the transfer resistors $\left(1,5.10^{-8}\right)$. A large amount of work has already been carried out to minimize the effect of the frequency dependence. In order to reduce the uncertainties associated to these bridges, a new generation of two-stage autotransformers has been developed and calibrated.

\section{Two-stage autotransformer standard}

\subsection{Principle}

An autotransformer is a transformer in which the secondary winding is a part of the primary one. Compared to a traditional transformer, it allows one to decrease the number of windings and avoids potential fluctuations between primary and secondary coils.

Two-stage autotransformers are currently used to provide standard ratios [5]. The primary winding of such a device is composed of two windings wound on two separate toroidal magnetic cores as shown on Figure 3. The magnetizing winding, is wound on core 1 , while the metrological winding, is wound around both the magnetic cores 1 and 2 . Since the metrological stage acts mainly to compensate the flux leakages and the magnetic losses in the magnetizing core, its section doesn't need to be as large as the magnetizing one. The metrological winding is divided into sections which allow one to define the bridge ratio.

Magnetizing and metrological windings have the same number of turns and are supplied by the same voltage source. This leads to a very low current in the metrological winding intended to provide the standard voltages. This induces a significant decrease of the effects of the leakage inductances and resistances which would exist in a single stage autotransformer. Therefore, the ratio corrections may be reduced by a factor of about $10^{3}$ compared to a single stage autotransformer, leading to a ratio correction value below $10^{-7}$ at $1 \mathrm{kHz}$.

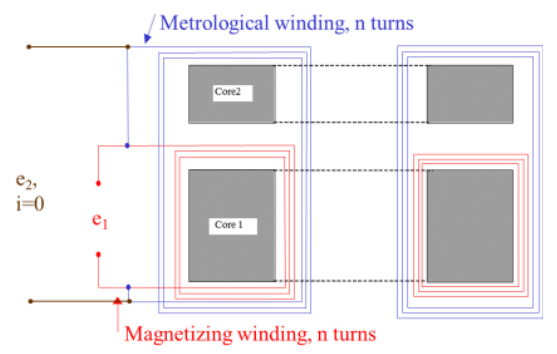

Figure 3. Sectional view of a two-stage autotransformer

\subsection{Magnetic core}

The magnetic cores are made of Nanophy ${ }^{\circledR}$ which has a very high permeability $\left(\mu_{\mathrm{r}}>100000\right.$ at $\left.1 \mathrm{kHz}\right)$ and a saturation flux density of $1.25 \mathrm{~T}$. They consist of a nonconductive thin strip (about $20 \mu \mathrm{m}$ ) tightly wound into a spiral which allows to reduce the influence of Eddy currents while maintaining an important total flux through the section of the core. The autotransformer is designed to work at voltages up to $240 \mathrm{~V}$ for frequencies between 400 and $1600 \mathrm{~Hz}$. Core sections are $19.5 \mathrm{~cm}^{2}$ for the magnetizing one and $4.5 \mathrm{~cm}^{2}$ for the metrological one.

\subsection{Windings}

Magnetizing and metrological windings have 240 turns. The magnetizing winding is made with enameled copper wire. Two guides (Figure 5 (a)) have been designed to obtain an uniform winding. An "anti-progression" turn has been added to decrease the effect of a disturbance due to a magnetic field perpendicular to the toroidal section [5].

The metrological winding has been wounded using a modified "Ayrton-Perry" method described in [5]. This method permits to decrease significantly the selfcapacitance of the winding and to make it insensitive to external magnetic fields. The winding consists of twelve sections of twenty turns each connected in series. They are made with three ribbons of four wires superimposed and separated by an insulator (Teflon). The potential allocation of the twelve wires has been chosen to minimize the effect of the inter-sections capacitances. Figure 4 shows the progression of application of the turns.

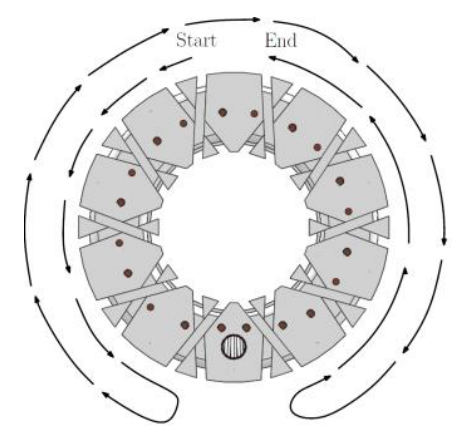


Figure 4. Progression of application of the turns using a modified "Ayrton-Perry" method

\subsection{Electric and magnetic screens}

Electric screen (Figure 5(b)) and magnetic screen (Figure $5(d)$ ) are placed between the magnetizing and metrological windings allowing, for the first, to eliminate capacitive currents between windings, and for the second, to reduce the magnetic leakage of the magnetizing stage. The assembly is placed in a second magnetic shield (Figure $5(\mathrm{c})$ ) to protect the autotransformer against external electromagnetic fields.

Electric screen is made of copper and magnetic screens are made of Mumetal ${ }^{\circledR}$.

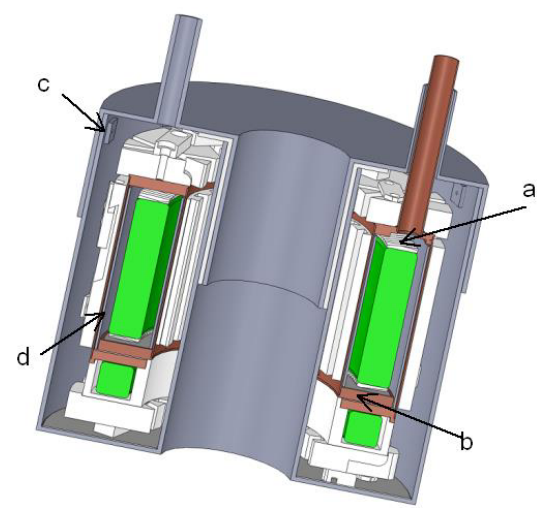

Figure 5. Sectional overview of the autotransformer

\subsection{Connectors}

The magnetizing stage is accessible by UHF connectors on the rear and the twelve sections (metrological stage) are accessible by BPO connectors on the front of the enclosing box as shown on Figure 6. Some of them were doubled to ensure the geometry's consistency of the measuring loop during calibration.
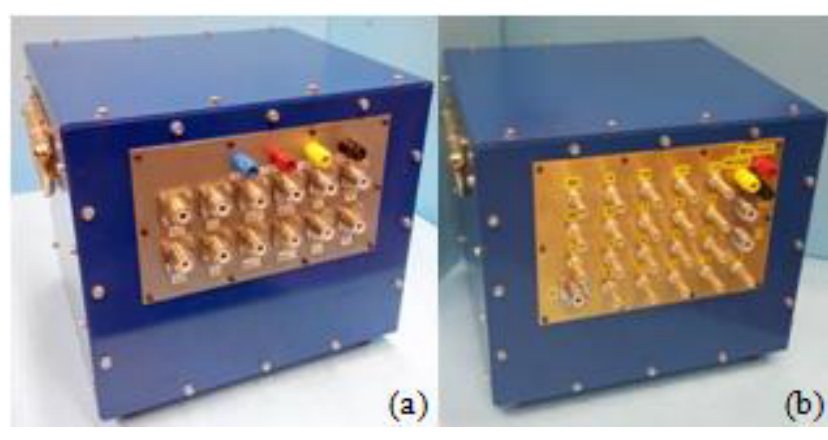

Figure 6. Final assembly of the standard two-stage autotransformer: (a) the rear, (b) the front

\section{Calibration}

The calibration is performed using the bootstrap method described in [5], slightly modified. It consists in successively comparing the terminal voltage of each section to a fixed voltage provided by a calibrating transformer. The voltage delivered by the calibrating transformer must be stable for the duration of the measurements and independent of the working potential relative to the ground potential. A dedicated two-stage calibrating transformer of ratios 1:11 or 1:12 (depending on the calibration configuration) was constructed for this purpose. The comparison is performed with no current in the detection loop. Balance is achieved by means of an injection transformer associated to a generator of adjustable voltage in phase and quadrature. The injection is made on the metrological stage of the calibrating transformer. The detection of the balance is performed using a detection transformer associated with a lock-in amplifier.

By this method, relative uncertainties on the ratio corrections can be reduced at a level down to a few parts in $10^{9}$ or better. Figure 7 shows the calibration bridge used for the characterization of the autotransformer.

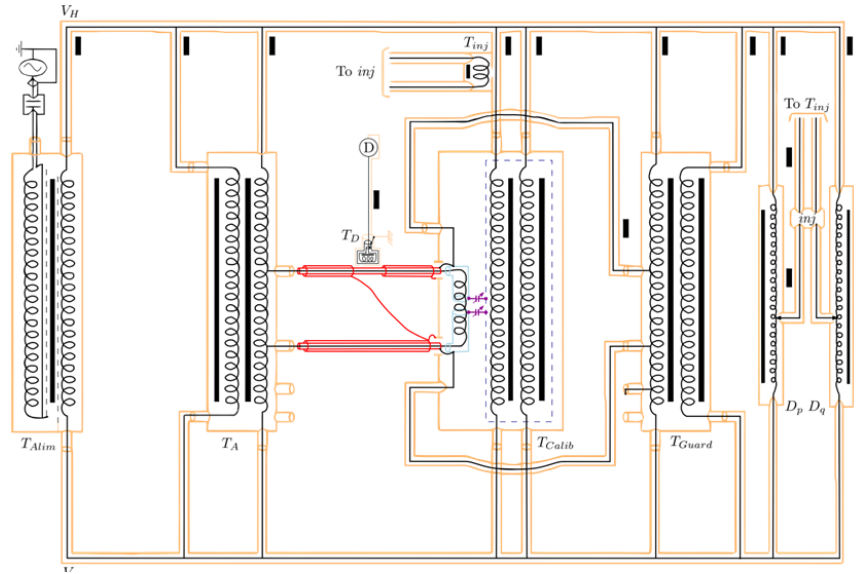

Figure 7. Calibration bridge with $T_{\text {Alim }}$ the supply transformer, $T_{A}$ the transformer under calibration, $T_{D}$ the detection transformer, $T_{i n j}$ the injection transformer, $T_{\text {Calib }}$ the calibrating transformer, $T_{\text {Guard }}$ the guarding transformer, $D_{p}$ and $D_{q}$ the inductive voltage dividers

\section{Results}

With this new generation of standard autotransformers, the corrections have been decreased compared to the previous autotransformer used during the last determination of $R_{\mathrm{K}}$. Those corrections have been determined with an uncertainty of about $1.10^{-9}$ from $400 \mathrm{~Hz}$ to $1600 \mathrm{~Hz}$ for voltages ranging from 10 to $200 \mathrm{~V}$. Hereafter are presented the corrections measured for the four frequencies at which the device is used.

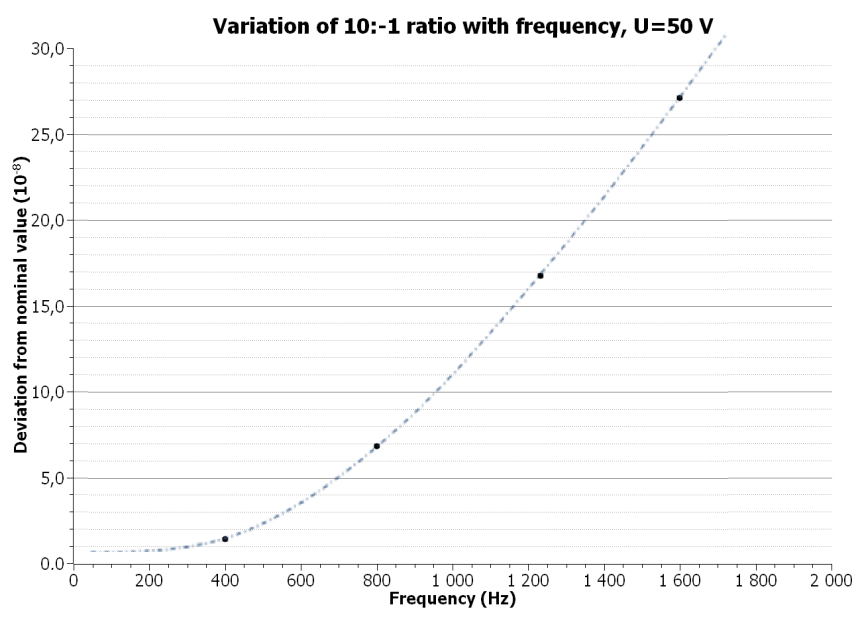


Figure 8. Variation of 10:-1 ratio with frequency at $50 \mathrm{~V}$. The quadratic behavior of the correction with frequency is explained in [5]

The voltage behavior has also been studied and no deviation larger than $2.10^{-9}$ are observed between $10 \mathrm{~V}$ and $200 \mathrm{~V}$ from $400 \mathrm{~Hz}$ to $1600 \mathrm{~Hz}$ instead of $3.10^{-8}$ between $10 \mathrm{~V}$ and $100 \mathrm{~V}$ for the last generation.

Figure 9 shows the variation of the correction with the voltage at $800 \mathrm{~Hz}$.

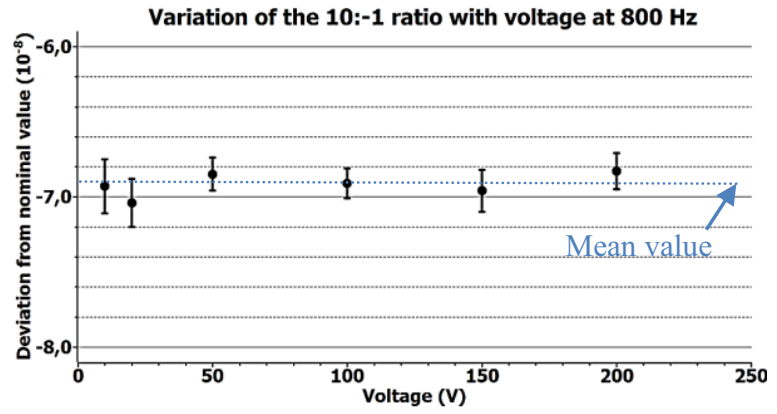

Figure 9. Variation of 10:-1 ratio with voltage at $800 \mathrm{~Hz}$

\section{Conclusion}

The design and construction of the standard two-stage autotransformer is now completed. The corrections have been decreased by a factor of three compared to the previous autotransformer at $1600 \mathrm{~Hz}$ and are known with an uncertainty of about $1.10^{-9}$ from $400 \mathrm{~Hz}$ to $1600 \mathrm{~Hz}$ for voltages varying from $10 \mathrm{~V}$ to $200 \mathrm{~V}$. The implementation of this autotransformer in the capacitance bridges used in the $R_{\mathrm{K}}$ measuring chain will reduce the contribution of the standard ratio uncertainty on the global uncertainty budget from 15 parts to 5 parts in $10^{9}$. This device is also used in the traceability chain implemented for the realization of the farad from the DC quantum Hall effect.

Furthermore; this new standard two-stage autotransformer could be used to determine the frequency behavior of the transfer resistances and the voltage effect of the transfer capacitors $10 \mathrm{pF}, 100 \mathrm{pF}, 1000 \mathrm{pF}$ in order to reduce the associated uncertainty components.

\section{References}

1. Resolution 1 of the $24^{\text {th }}$ meeting of the CGPM, BIPM Web site, http://www.bipm.org/eu/CGPM/db/ 24/1/,2011.

2. G. Trapon, O. Thévenot, J.C. Lacueille, W. Poirier, "Determination of the von Klitzing constant $R_{\mathrm{K}}$ in terms of the BNM calculable capacitor - fifteen years of investigations", Metrologia, 40, Iss. 4, 159171 (2003).

3. P.Gournay, O.Thevenot, L.Dupont, J.M. David, F.Piquemal, "Toward a determination of the fine structure constant at LNE by means of a new
Thompson-Lampard calculable capacitor", Can. J. of Physics, Vol. 89, $\mathrm{n}^{\circ} 1,169,2011$

4. D. Cutkosky, "Techniques for comparing FourTerminal-Pair admittance standards", Journal of Research of the NBS, Vol. 74C, No 3 and 4, p.63, (1970).

5. B.P. Kibble, G.H. Rayner, "Coaxial AC Bridges", Ed. A. E. Bailey, (1984). 